\title{
Titre : L'écologie industrielle et territoriale : une politique alternative pour la gestion de la décroissance urbaine? Le cas de Dunkerque
}

\author{
Christophe Beaurain ${ }^{1}$, Chedrak De Rocher Chembessi ${ }^{2}$, \\ 1 Professeur d'économie-aménagement, Université de Limoges, GEOLAB UMR 6042, \\ christophe.beaurain@unilim.fr \\ 2 Doctorant, Université de Limoges, GEOLAB UMR 6042 - Université Laval, Centre de \\ Recherche en Aménagement du Territoire et Développement Régional, \\ chedrak.chembessi@unilim.fr / chedrak-sylvain-de-rocher.chembessi.1@ulaval.ca
}

\section{Résumé :}

L'émergence récente de la problématique des villes en décroissance en France met en lumière différentes politiques publiques s'inscrivant dans une logique d'attractivité. Cependant, les recherches sur la ville décroissante, notamment en France, n'abordent que très peu la question de la transition écologique sur laquelle pourrait reposer leur re-développement. S'appuyant sur une lecture de la décroissance urbaine qui tire ses fondements dans le vaste courant de pensée sur l'utopie urbaine d'une part, et les enjeux de transition écologique d'autre part, cet article a pour but d'esquisser une réflexion sur les potentialités offertes à ces territoires en déclin par la mise en œuvre de stratégies alternatives de gestion des ressources territoriales. Il s'agit, au travers des synergies industrielles de Dunkerque (France), de définir les apports d'un changement de paradigme dans les organisations productives de la ville en décroissance à la construction d'un projet alternatif urbain.

Mots Clefs : écologie industrielle et territoriale - décroissance urbaine - transition écologique - action collective

\begin{abstract}
:
In France, recent researches on « shrinking cities » outline various local public policies which have been implemented for a new attractiveness of these urban areas. However, these studies do not address too much the ecological transition in this urban regeneration. In fact, this article aims to outline potentials contributions and impacts of alternative strategies of local resources management by firms. In order to measure these impacts, we have contextualized an urban shrinkage thought urban utopia and ecological transition. In addition, we have used for empirical analysis data from city of Dunkerque (France) where various industrial ecology
\end{abstract}


projects have been implemented since many years. With these both approaches, this paper demonstrates how transition can emerge as alternative policy of urban development.

Keys Words: industrial ecology - urban shrinkage - ecological transition - collective action 


\section{Introduction}

Durant le vingtième siècle, le récit de l'urbanisation s'est construit autour des incidences positives de la localisation des activités économiques, de la croissance industrielle et tertiaire, consubstantielles à la concentration de nouvelles populations en milieu urbain (Bost, 2014). Cependant, les crises économiques successives qui ont frappé de nombreux secteurs d'activités (mine, industrie, immobilier, finance, etc.) depuis les années 1970, plus particulièrement aux États-Unis et en Europe occidentale, ont mis en évidence les difficultés de certaines villes en proie à des phénomènes de déclin démographique et industriel, et plus globalement de décroissance urbaine (Wolff et al., 2013).

Cette double mutation urbaine ne se limite pas à d'anciennes villes industrielles. En effet, des petites villes ou des villes secondaires, caractérisées par l'importance de l'emploi public et du tertiaire à faible valeur ajoutée, font elles aussi l'expérience d'un déclin autant en France que dans le reste du monde (Paulus, 2004; Wolff et al., 2013). Dans ce contexte, la mesure du déclin urbain, et plus généralement sa caractérisation socio-économique, fait l'objet depuis quelques années de recherches approfondies, à partir de cas américains, britanniques ou français notamment (Béal, Fol, \& Rousseau, 2016; Fol \& Cunningham-Sabot, 2010; Rousseau \& Béal, 2015). Il y est en particulier question d'analyser les politiques publiques menées dans ces contextes de décroissance urbaine, en soulignant dans la plupart des cas la persistance du retour à la croissance comme issue incontournable au déclin des villes.

Dans cet article, nous proposons une réflexion sur les contours d'un modèle productif alternatif associé à ces configurations de ville en décroissance. Plus précisément, nous mettons l'accent sur les apports potentiels d'un engagement collectif des acteurs de ces espaces urbains dans une démarche de transition écologique. Ce faisant, notre réflexion propose une remise en cause des politiques publiques classiques de retour à la croissance économique par la prise en compte des contraintes environnementales vis-à-vis des processus de production. Nous soulignons ici la contribution possible des démarches d'écologie industrielle et territoriale comme dynamiques alternatives, associant l'ensemble des acteurs du territoire. Nous abordons brièvement la réflexion émergente autour de la " valeur territoriale » portée par les modèles soutenables (Maillefert \& Robert, 2017), en la confrontant au contexte de la décroissance urbaine. Pour ce faire, nous développons un argumentaire en trois temps.

En premier lieu, nous essayons de resituer brièvement les enjeux de cette problématique des villes en décroissance au regard des théories de la décroissance et de l'histoire des projets urbains. Nous rappelons, ensuite, les principes fondamentaux du changement de paradigme amenant à l'écologie industrielle et territoriale, saisie à travers l'engagement d'une action collective et multiscalaire (Beaurain \& Varlet, 2015). Enfin, dans une dernière partie, nous mettons en avant les contributions de l'écologie industrielle et territoriale sous un double point de vue porteur selon nous d'un potentiel de politiques alternatives : l'évolution urbaine vers la permacircularité et la soumission de la croissance aux contraintes environnementales qu'elle implique (Arnsperger \& Bourg, 2017) d'une part ; la confrontation des multiples valeurs associées à la dynamique économique en milieu urbain et la réflexion sur les 
fondements d'une action collective, d'autre part. Nous concluons alors sur les perspectives qu'offre potentiellement une dynamique locale de transition écologique, non pas en termes de retour à la croissance et de création d'emplois dans les secteurs traditionnels de l'économie mais plutôt en termes d'émergence, au sein de ces villes en décroissance, d'un nouveau modèle de développement incluant l'ensemble des populations.

Notre recherche s'appuie principalement sur des projets d'écologie industrielle et territoriale mis en œuvre depuis quelques années au sein de l'agglomération de Dunkerque (France). Ces initiatives territoriales associent des acteurs privés (synergies industrielles) et publics (réseau de chauffage urbain, et actions de régulation des interactions entre entreprises privées) dans le cadre d'une stratégie urbaine de redéveloppement ${ }^{3}$.

\section{Utopie urbaine et décroissance : regards croisés}

\subsection{L'histoire de l'utopie urbaine peut-elle nous aider pour la compréhension des villes en décroissance?}

Si l'émergence de la problématique des villes en décroissance est liée aux récentes crises dans les pays industrialisés, la réflexion qu'elle suscite peut néanmoins être brièvement replacée dans une histoire plus ancienne. La difficulté à penser l'articulation entre le projet urbain et la critique du capitalisme est en effet récurrente dans l'histoire de la réflexion sur l'utopie urbaine depuis le $19^{\text {ème }}$ siècle, particulièrement celle qui s'est focalisée sur les rapports entre ville et nature (Beaurain \& Andrieu, 2016). Ainsi, les argumentaires pionniers des utopistes du $19^{\text {ème }}$ siècle, ou ceux moins radicaux au début du $20^{\text {ème }}$ siècle se sont intéressés à la question de la limitation de la taille urbaine et à l'importance du rapport de l'habitat urbain à l'expérience du contact à la nature (Howard, 1902; Geddes, 1915). Mais, la question économique n'est restée que très marginalement abordée. Le vingtième siècle aura été celui du grand récit des effets positifs conjoints de la croissance économique et urbaine.

Dans ce contexte, l'œuvre prolifique de Lewis Mumford constitue à bien des égards une tentative particulièrement érudite de critique du capitalisme et de la puissance destructrice de la technique. Sa condamnation de la métropolisation et sa dénonciation de la «cité carbonifère " du $19^{\text {ème }}$ siècle et des métropoles de l'après seconde guerre mondiale s'inscrivent en effet dans une remise en cause de l'illusion techniciste propre au capitalisme et dans une volonté de s'interroger sur le retour nécessaire à une taille urbaine plus propice à la prise en compte de l'environnement (Mumford, 1964, 2016). Plus récemment, des argumentaires radicaux comme ceux d'Alberto Magnaghi (2003, 2014) de Rob Hopkins (2010), ou encore de North et Nurse (2014) critiquent également l'obsession de l'articulation entre croissance économique et urbaine, en rattachant leurs réflexions aux problématiques

\footnotetext{
${ }^{3}$ Pour l'analyse de ces projets d'écologie industrielle, les données ont été recueillies dans le cadre de précédents travaux (Beaurain, Varlet; 2015; Beaurain, Maillefert, Varlet, 2017) à partir d'entretiens semi-directifs effectués auprès de dirigeants d'entreprise ou responsables environnement de l'agglomération dunkerquoise engagés dans ces projets et représentatifs des principaux flux de la symbiose industrielle dunkerquoise. Une vingtaine d'entreprises et d'acteurs institutionnels locaux avaient alors été interviewés. Nous avons complété ces données qualitatives par des données quantitatives de la situation socio-économique de cette agglomération.
} 
environnementales planétaires apparues depuis quelques années (réchauffement climatique, épuisement des ressources naturelles...). Ces argumentaires associent explicitement le projet urbain ou territorial à la définition d'un modèle de développement de l'après-capitalisme. Néanmoins, ils restent silencieux sur le chemin à parcourir pour sortir de l'ère du capitalisme, notamment au regard des contraintes nouvelles sur les enjeux environnementaux par exemple.

\subsection{La notion de « décroissance » a-t-elle un sens ?}

Depuis la deuxième moitié du vingtième siècle, des argumentaires critiques du capitalisme ont associé la décroissance ou l'état stationnaire tantôt aux limites intrinsèques du capitalisme du point de vue de la consommation énergétique et des prélèvements de matières premières (Daly \& Farley, 2010; Jackson, 2009; Meadows \& Randers, 2012), tantôt à une mise en évidence des limites économiques du capitalisme et de son remplacement inéluctable par un autre modèle économique (Gadrey, 2011; Gorz, 2008). Ces approches constituent un cadre théorique potentiellement intéressant pour les perspectives d'évolution des villes en décroissance, dans un objectif autre que le retour à la croissance. Cependant, cette notion de décroissance doit, de l'aveu même de certains de ces auteurs, être utilisée avec beaucoup plus de précautions qu'elle ne le fut à la fin du siècle précédent (Clerc, 2004; Montel, 2017) ${ }^{4}$. De plus, aussi intéressantes soient-elles, ces approches n'ont que très marginalement intégré la dimension urbaine et les politiques qui la structurent. Et cela laisse planer l'hypothèse d'une décroissance déterritorialisée, à traiter uniquement au niveau macro-économique. Cependant, la problématique des villes en décroissance, quant à elle, renvoie à une lecture plutôt territorialisée, c'est-à-dire à la nécessité de considérer les actions locales menées par les acteurs des territoires. En redéfinissant un ancrage territorial au sein du cadre théorique de la décroissance urbaine, il apparaît plus aisé d'aborder différentes questions, notamment relatives aux stratégies alternatives de développement économique.

\subsection{Des politiques alternatives dans les villes en décroissance}

Est-il possible d'articuler le projet urbain à un modèle territorialisé de développement économique, et pouvant s'inscrire dans un contexte de décroissance ? À défaut de pouvoir répondre par l'affirmative à cette question, les interrogations actuelles sur la transition écologique offrent des pistes de réflexion intéressantes, notamment à travers les questions posées aux politiques de régénération urbaine. En effet, la question écologique interpelle à la fois la transformation nécessaire des modes de production et de consommation, mais aussi les perceptions sociales et individuelles de l'importance du rapport à la nature. Cela suppose que la décroissance urbaine puisse être placée au cœur d'une réflexion systémique répondant à un besoin de (ré)attractivité du territoire au travers de différentes formes d'innovation socioéconomique (agricultures urbaines, systèmes d'échanges locaux, systèmes alimentaires territorialisés, démocratie participative...). Pour cela, nous nous inscrivons dans la perspective tracée par certains auteurs, qui soulignent que la décroissance urbaine doit être considérée

\footnotetext{
${ }^{4}$ En fait, pour beaucoup d'auteurs tenants de la « décroissance », celle-ci signifie davantage une critique radicale de la croissance comme horizon indépassable des politiques publiques qu'un appel à s'engager dans le chemin de la décroissance du PIB, chemin guère praticable en réalité.
} 
sous le prisme d'un potentiel de stratégies alternatives, aidant à répondre à des impératifs écologiques et sociaux contemporains (Béal \& Rousseau, 2014; Harvey, 2004). Cependant, le dogme qui fait des entreprises le cœur des stratégies collectives de sortie de crise et/ou d'attractivité reste fort présent dans le volontarisme politique des acteurs publics locaux.

Quant au potentiel alternatif, il s'appuie au contraire sur des politiques « bottom-up », sur l'expression de multiples valeurs non nécessairement marchandes, et la défense d'intérêts des plus défavorisés (Béal \& Rousseau, 2014). Ce potentiel ouvre ainsi les perspectives d'un développement territorial, plus égalitaire et plus convivial, dans lequel la prospérité n'est pas reliée à la consommation mais au bien être humain défini dans une situation territoriale spécifique par les citoyens eux-mêmes (North \& Nurse, 2014).

Si l'on considère que ces initiatives renvoient fondamentalement à la construction d'une action collective engageant l'ensemble des acteurs d'un territoire, il apparaît indispensable d'interroger le rôle des entreprises dans ce potentiel transformatif, sans se limiter aux pratiques des grandes firmes mondialisées qui sont souvent mues prioritairement par la croissance de la valeur actionnariale. De ce point de vue, face au poids croissant pris depuis quelques années par les marchés financiers et les attentes des actionnaires en termes de dividendes sur la gouvernance des firmes, la dimension collective des entreprises est à considérer à travers leur participation à la construction de nouveaux potentiels de création de valeur s'intégrant dans un progrès collectif de la société (Hatchuel \& Segrestin, 2007). A travers un nouveau statut, il s'agirait ainsi de reconnaître à l'entreprise une véritable « mission » de transformation sociale, par son engagement vis-à-vis de l'ensemble de la société dans le cadre d'un "agir responsable» (Hatchuel, Segrestin, 2016 ; Hatchuel, Levillain, Segrestin, 2014).

Soulignons enfin le rôle clé des situations de crise (économique, démographique, écologique...) dans l'émergence et la pérennisation du potentiel alternatif (Béal \& Rousseau, 2014). North et Nurse affirment ainsi que la lutte contre le changement climatique constitue un puissant vecteur de remise en cause des politiques libérales de compétitivité territoriale. Plus spécifiquement, l'enjeu de transition écologique et énergétique offre des opportunités pour une mutation des systèmes productifs et l'émergence d'un paradigme socioéconomique alternatif, se construisant à partir de dispositifs institutionnels et d'actions collectives territorialisées. Plus précisément, nous pensons que les dynamiques impulsées dans ce domaine par les démarches d'écologie industrielle et territoriale, et plus récemment d'économie circulaire, représentent un réel potentiel transformatif des systèmes productifs locaux, dans le contexte socioéconomique des villes en décroissance, notamment en ce qui concerne la consommation de l'énergie et des ressources naturelles.

\section{Les perspectives de l'écologie industrielle et territoriale comme stratégie alternative}

\subsection{Les principes fondamentaux de l'écologie industrielle et territoriale}

La volonté de dépasser un modèle d'économie linéaire à l'origine de bon nombre d'impacts environnementaux s'illustre ces dernières années par un changement dans les pratiques socio- 
environnementales des entreprises (Lilley, 2009; Preston, 2012). De manière singulière, l'écologie industrielle et territoriale s'appuie sur un ensemble de modalités pour un développement moins dépendant de la consommation des ressources, au travers d'une approche écologique des activités économiques (Erkman, 2004). Dans un objectif de découplage de la croissance économique de la consommation des ressources, l'écologie industrielle et territoriale renvoie à une transformation du modèle productif en un modèle plus intégré, fonctionnant de manière similaire à un écosystème biologique (Allenby, 1992). Ce modèle intégré permettrait de considérer les déchets et/ou les sous-produits générés par les activités industrielles comme de véritables ressources, à l'instar des symbioses biologiques, en dépassant les limites de l'approche « end of pipe»(Erkman, 1997), pour tendre finalement vers une économie sobre. Il s'agit, entre autres, d'une remise en cause fondamentale du modèle « linéaire » ayant caractérisé le développement industriel antérieur où «les flux de matières d'un stade à l'autre sont indépendants de tous les autres flux », générant ainsi des émissions importantes de polluants et de déchets à tous les stades de la production (Jelinski et al., 1992). Cette remise en cause doit être considérée à la fois d'un point de vue technique mais aussi des relations interentreprises. Sa mise en œuvre dépasse ainsi le strict cadre des flux techniques, en tenant compte également des questions de coordination marchande, structurelle et organisationnelle (Ehrenfeld, 2004; Korhonen, 2004). Elle renvoie davantage à un projet de territoire s'intéressant à un ensemble des flux territoriaux d'une part, et associant à une pluralité d'acteurs territoriaux d'autre part.

\subsection{L'inscription de l'ÉIT dans l'économie circulaire et la transition écologique}

Depuis une dizaine d'années maintenant, les démarches d'ÉIT s'inscrivent dans une vision plus globale de la transformation écologique et sociale à différentes échelles, plus précisément au niveau des produits et/ou procédés industriels, au sein des filières et/ou des territoires, et à des échelles macro-sociétales. De ce point de vue, la référence à l'économie circulaire offre l'avantage d'insérer l'ÉIT dans une approche nationale et institutionnelle s'appuyant sur un cadre législatif et une vision économique de la gestion des ressources (Buclet, 2015) . Selon $^{5}$ la définition de l'Ademe $^{6}$, l'économie circulaire doit être appréhendée à partir de trois domaines d'action et de sept piliers constitutifs de ces domaines: l'offre des acteurs économiques (ÉIT, l'approvisionnement durable, l'éco-conception, l'économie de la fonctionnalité), la demande et le comportement des consommateurs (allongement de la durée d'usage avec les 3R, consommation responsable), et la gestion des déchets (recyclage) (Ademe, 2017).

\footnotetext{
${ }^{5}$ En la matière, il faut souligner le rôle pionnier de la Chine à partir de 2005 avec la succession depuis cette date de documents définissant une stratégie de développement de l'économie circulaire dans le cadre des différents plans quinquennaux, l'édiction d'une loi sur la promotion de l'économie circulaire à l'hiver 2008, et la construction d'indicateurs permettant de mesurer l'évolution des politiques en ce domaine, notamment au niveau des villes (Aurez, Georgeault, 2016a ; 2016b). En France, les objectifs associés au développement de l'économie circulaire ont été intégrés dans la «stratégie nationale de transition écologique pour le développement durable 2015-2020» (axe2) et dans la loi de transition énergétique pour la croissance verte (TEPCV) en 2015.

${ }^{6}$ Selon l'Ademe, «l'économie circulaire peut se définir comme un système économique d'échange et de production qui, à tous les stades du cycle de vie des produits (biens et services), vise à augmenter l'efficacité de l'utilisation des ressources et à diminuer l'impact sur l'environnement tout en développant le bien être des individus » (Ademe, 2017).
} 
$\mathrm{Si}$, dans les faits, les aspects liés au réemploi, à la réparation et à l'éco-conception constituent pleinement des leviers d'opérationnalisation de l'économie circulaire, ils sont encore souvent considérés de manière isolée au détriment de la vision systémique largement défendue. Ainsi, l'économie circulaire reste encore cantonnée au recyclage et à la valorisation, et aux relations inter-entreprises qui se définissent par des synergies de substitution et de mutualisation de flux, construites sur les principes de l'écologie industrielle (Hobson, 2016; Lyle, 1994). Cette situation tend à masquer le fait que l'économie circulaire englobe un ensemble de stratégies, outre le recyclage, permettant de générer de nouveaux modèles d'affaires capables de conduire à un développement plus soutenable (Ghisellini, Cialani, \& Ulgiati, 2016). Elle dépend ainsi de la mobilisation d'un réseau d'acteurs locaux, dans une dynamique relationnelle et collaborative. Le fonctionnement en boucle fermée de l'économie qui est promu - si on s'en tient au seul aspect de synergies de flux - doit nécessairement s'appuyer sur une approche systémique faisant interagir différents acteurs territoriaux. De plus, le territoire est saisi comme un centre de création de valeur à travers sa dimension multifonctionnelle (Maillefert \& Robert, 2017), constituant dès lors un levier important dans le développement de l'économie circulaire. Cependant, la fixation sur la valeur ou la captation d'un avantage concurrentiel autant individuellement que collectivement donne amplement du sens aux questionnements ou autres critiques «d'une démarche de reproduction et/ou de survie de la logique capitalistique » (Arnsperger \& Bourg, 2017; Harvey, 2010). Dès lors, la valeur à la fois économique, sociale et environnementale que l'économie circulaire produit ne devrait plus être limitée à la seule entreprise, mais devrait constituer une valeur partagée par un ensemble de parties prenantes (Bocken et al., 2014), voire au service d'un processus de développement territorial (Beaurain \& Brullot, 2011).

Si l'ÉIT apparaît donc comme une dimension constitutive essentielle de l'économie circulaire, elle doit être également intégrée dans l'institutionnalisation croissante de la transition écologique, ne serait-ce que du point de vue de l'enjeu de sobriété dans la consommation des ressources et de l'énergie. Encore ne faut-il pas réduire la transition écologique à une simple adaptation des processus économiques à des objectifs de durabilité plus ou moins flous. La transition écologique doit en effet être associée à une véritable réflexion sur l'adaptation de ces processus à la finitude des ressources et sur le développement d'une démocratie écologique (Bourg, 2012). Dans cette logique, elle renvoie également à l'impulsion donnée aux initiatives des citoyens et de l'ensemble des acteurs comme reflets d'une expérimentation sociale, à une transformation en profondeur de l'action publique s'articulant aux initiatives citoyennes, et à une relocalisation de certaines activités économiques (Laigle, 2013 ; Angot, 2013). À titre d'exemple, l'enjeu énergétique de la transition écologique fait partie intégrante des démarches d'écologie industrielle. La dimension énergétique de l'ÉIT se construit en effet autour de la récupération et la redistribution des flux d'énergie et de chaleur issus des processus industriels et de l'incinération des déchets, ou à travers les activités de méthanisation issus des relations entre agriculture et industrie. Elle contribue ainsi potentiellement à la relocalisation de la production énergétique, en se fondant sur la proximité géographique des producteurs et consommateurs concernés. Le domaine du chauffage urbain, 
et particulièrement celui des bâtiments à usage collectif, se présente comme une application phare de cette récupération de source d'énergie. En effet, la transition énergétique est synonyme de reconnexion entre les lieux de production et de consommation, débouchant potentiellement sur une remise en cause de la logique réticulaire des infrastructures de distribution de l'énergie (Hampikian, 2017). Ainsi, des changements se dessinent au travers d'activités de recyclage et de récupération des énergies et de productions décentralisées de celles-ci dans des formes diverses (Raineau, 2011). Dans ce tournant, la relocalisation de la chaîne industrielle de production énergétique offre une occasion pour les territoires de construire des modalités spécifiques d'accès à l'énergie et un pilotage multi-acteurs de la gouvernance énergétique (Rocher, 2013).

En définitive, l'ÉIT comme dimension constitutive de l'économie circulaire renvoie aux capacités d'actions des territoires pour réduire localement les charges écologiques et environnementales. Néanmoins, elle ne peut être réellement associée à cet objectif qu'à la condition qu'elle s'insère effectivement dans une démarche de transition écologique répondant notamment aux impératifs de sobriété dans la consommation des ressources. C'est dans ce cadre que l'ÉIT doit alors s'insérer dans un véritable projet de territoire qui aille audelà d'une simple logique de verdissement de l'économie. Son approche doit consister à orienter autant que possible la dynamique économique vers des activités et des emplois compatibles avec la réduction de l'empreinte écologique.

\subsection{De la démarche d'écologie industrielle et territoriale de Dunkerque.}

Devenue une référence française en la matière, la démarche d'écologie industrielle et territoriale de Dunkerque illustre à la fois l'importance des échanges matériels et immatériels, et la complexité des interdépendances, entre les entreprises d'une part, entre les entreprises (ou autres acteurs locaux) et le territoire d'autre part, (Beaurain \& Varlet, 2015; Varlet, 2012).

\section{Dunkerque : une décroissance urbaine difficile à mesurer?}

La Communauté Urbaine de Dunkerque (CUD), créée en 1968 et qui regroupe aujourd'hui 19 communes, a connu depuis la fin des années 1950 d'importantes évolutions socioéconomiques. En effet, le choix de l'État français d'y installer une unité de sidérurgie « sur l'eau » à la fin des années 1950 a impulsé une forte croissance de l'activité industrielle jusque dans les années 1980. Conséquemment, l'arrivée d'Usinor a entrainé un développement très important des infrastructures urbaines. D'autres implantations industrielles vont assez vite se multiplier et différentes opérations d'urbanisme (ZUP), sur le territoire des communes rurales de Grande-Synthe et Petite-Synthe vont permettre aux populations accédant à ces nouveaux emplois industriels, généralement peu qualifiés, de bénéficier de logements décents, quoique construits dans l'urgence. Ce regain d'activité industrielle a par ailleurs bénéficié de la présence d'une plateforme portuaire avec ses chantiers navals, et d'importantes dynamiques transfrontalières entre la France, l'Angleterre et la Belgique. 
Au plan démographique, la période intercensitaire de 1962-1975 correspond à une période de forte croissance pour l'actuelle CUD, avec un accroissement de 4,7\% par an. Cette croissance démographique a surtout concerné les communes proches des implantations industrielles (Joan et allii, 2017). La carte 1 témoigne d'une hausse significative des effectifs de population dans les communes locales à l'exception de Saint Georges-sur-l'Aa. Commune rurale à l'extrémité de la communauté urbaine, celle-ci a sans doute été touchée par des mouvements de populations, préférant s'installer à proximité des zones industrielles. Les communes du littoral, telles que Grande-Synthe, Dunkerque, Fort-Mardyck, Loon-Plage ont été les grands bénéficiaires de cet afflux de population.

\section{Carte 1 : Taux de croissance démographique entre 1968 et 1982}

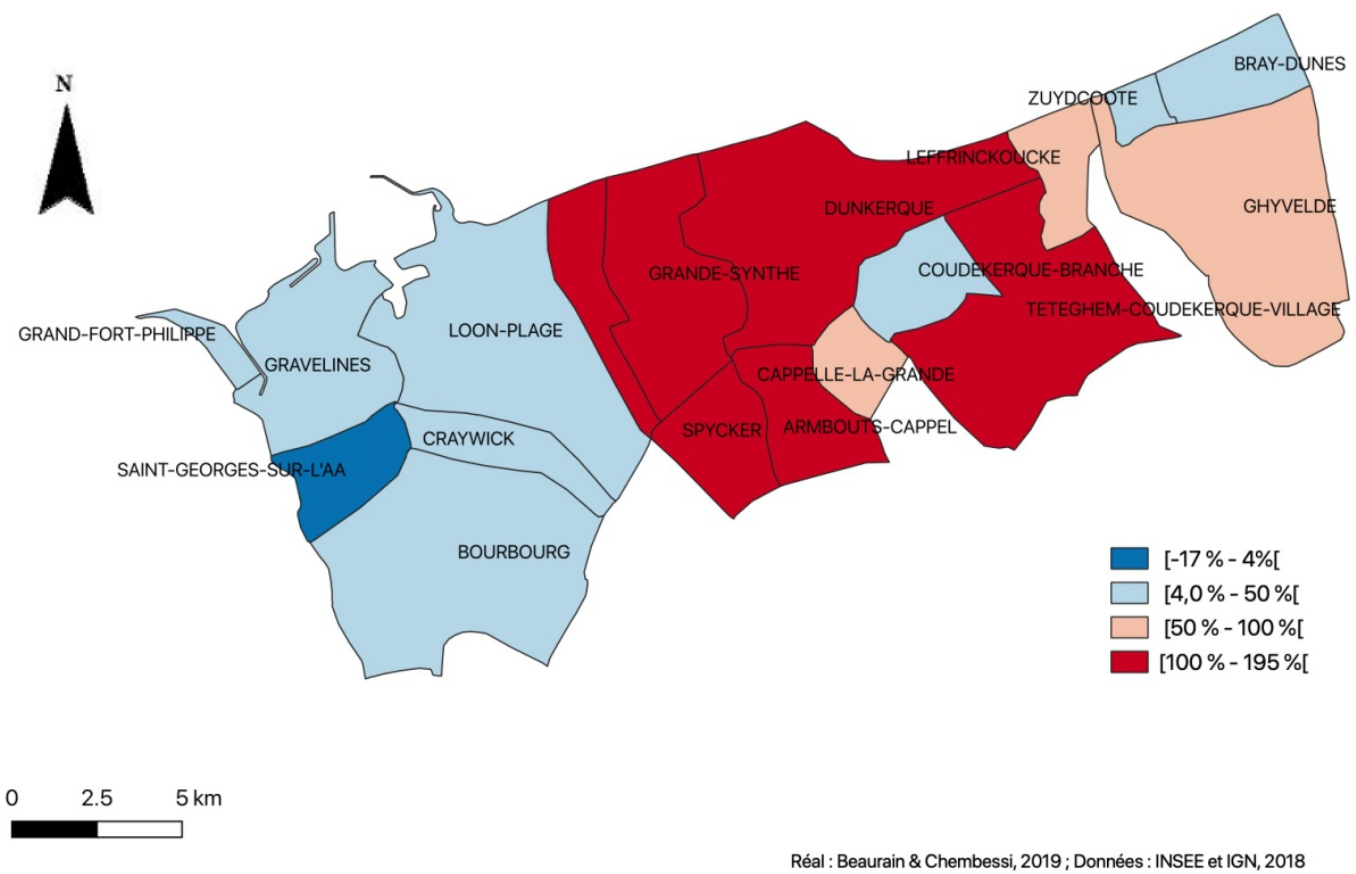

Cette croissance de l'activité industrielle a aussi permis à certaines communes de connaître des baisses considérables du taux de chômage et de maintenir celui-ci à environ $2 \%$ dans les années 1960 à 1970, taux faible comparativement au reste de la France. La structure politique, sociale et économique de Dunkerque reposait alors sur la dynamique impulsée notamment par le Port de Dunkerque et le complexe sidérurgique Usinor, devenu depuis Arcelor Mittal (Clochey, 2017). L'implantation de la sidérurgie a complètement façonné une nouvelle configuration urbaine (Castell, Godard, 1974) : la structuration socio-spatiale de la ville a été profondément transformée, avec notamment la création d'une agglomération intégrant les zones industrielles et l'extension de la zone portuaire au-delà des limites traditionnelles de la ville. Cette dépendance à l'égard de l'activité industrialo-portuaire a été, en partie, à l'origine des crises qui ont frappé par la suite l'agglomération. 
En effet, à partir des années 1980, les crises économiques successives ont révélé les fragilités d'une telle évolution socioéconomique: chute drastique des effectifs industriels, déclin démographique pour les communes proches des industries, dégradation d'un habitat construit trop rapidement, détérioration progressive des commerces de centre-ville... Ce déclin économique ne saurait néanmoins être interprété de manière univoque comme un rétrécissement de la ville au regard des théories de la décroissance urbaine. Si les communes industrielles ont effectivement connu un marasme économique, avec pour conséquence une faible attractivité, cette évolution s'est accompagnée d'un étalement urbain et d'un développement des petites communes rurales, ce qui a permis dans une certaine mesure de contenir la manifestation d'un phénomène de décroissance urbaine à l'échelle de l'agglomération. L'étalement urbain de Dunkerque - perçu ici par des gains de population dans des communes hors zones industrielles - qui s'est accentué après les crises des années 1980, rejoint l'illustration du phénomène de décroissance urbaine comme "un éclatement spatial des villes sous l'effet de la périurbanisation »(Charmes, 2011). L'impact de la réduction de l'activité industrielle sur la ville-centre ne peut néanmoins être contesté. Au moins huit communes situées en périphérie des centres industriels ont connu de véritables gains de population. Cette croissance périurbaine peut être associée à une forme de dévitalisation de la commune principale. Cette difficulté à mesurer convenablement la décroissance urbaine à Dunkerque existe aussi pour beaucoup d'autres aires urbaines françaises concernées par le même phénomène (Wolff et al., 2013). Le déclin démographique ne se traduit pas de la même manière entre les communes à l'échelle d'une structure intercommunale. Dans le cas de Dunkerque, la perte de population a davantage touché les communes disposant d'importants actifs industriels et économiques, notamment GrandeSynthe et Dunkerque, qui accueillaient une large part de la population ouvrière. Les autres communes proches des zones industrielles ont connu une stagnation voire un léger déclin démographique tandis que la croissance de la population se reportait sur les petites communes rurales plus éloignées de l'industrie (Joan et al., 2017).

\section{Carte 2 : Taux de croissance démographique entre 1982 et 2000}




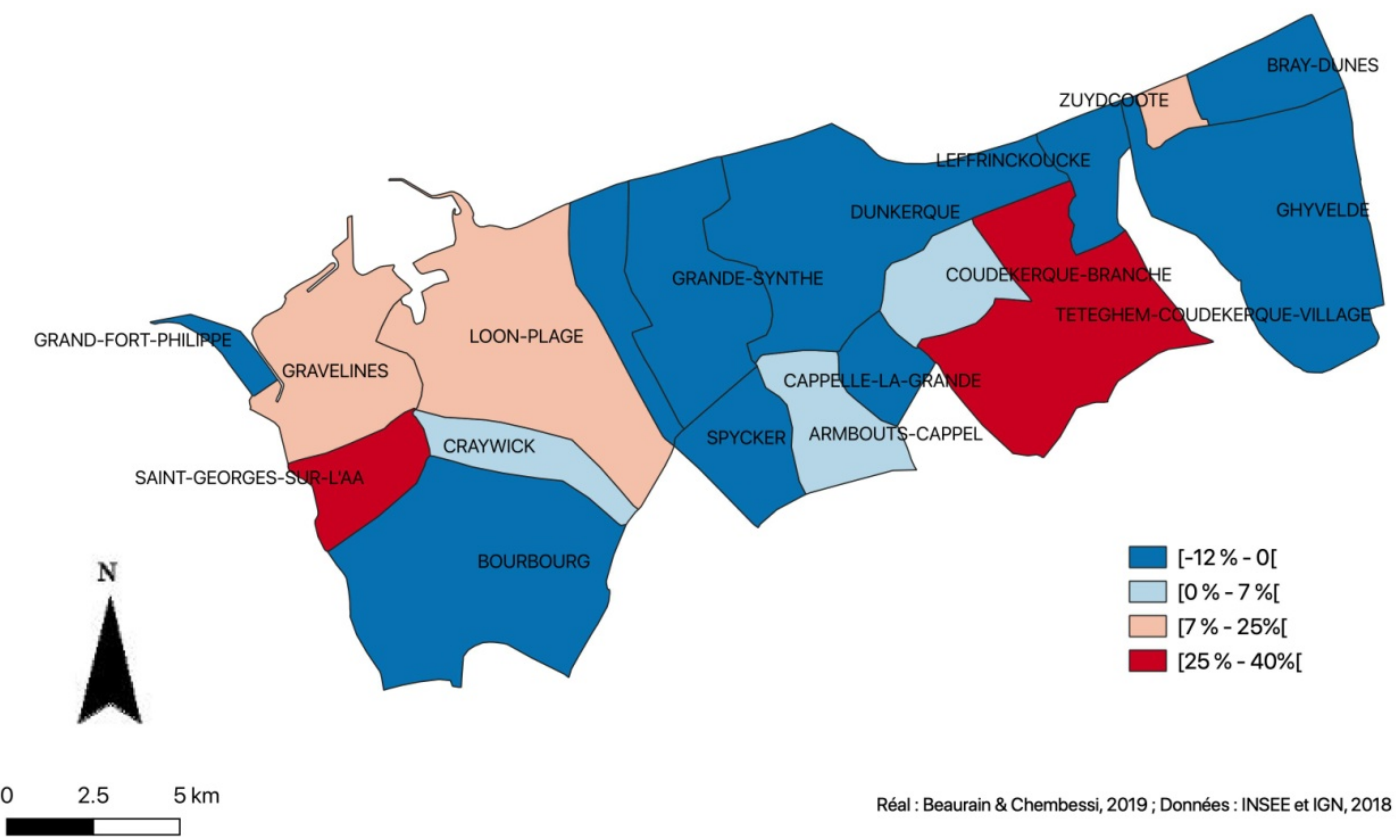

En effet, même si Dunkerque ne peut être considérée stricto sensu comme une ville en décroissance, l'agglomération présente bien des caractéristiques justifiant cette comparaison. Si l'on considère que la décroissance urbaine possède une manifestation plurielle, l'histoire urbaine de Dunkerque, dans le rapport entre villes et industries, s'inscrit dans une configuration proche des villes en décroissance. En plus de la dimension démographique, on peut aussi ajouter la baisse des actifs économiques, corroborée par une diversification économique moyenne. L'effectif d'emplois salariés dans le secteur privé (hors agriculture) connait une diminution considérable dans la zone d'emploi de Dunkerque, avec un taux de chômage supérieur à la moyenne nationale d'au moins 3 points ${ }^{7}$. Cette hausse du chômage est aussi à nuancer par des disparités entre les communes (Carte 3). La chute de l'emploi industriel a été en partie contrecarrée par l'emploi public et le développement d'une économie de services. Cependant, la croissance de l'emploi tertiaire est encore très timide. Et globalement, l'attractivité économique du bassin d'emploi reste encore à ce jour très limitée, notamment pour une main d'œuvre qualifiée.

\section{Carte 3 : Évolution du taux de chômage de 1982 à 1999 (en \%)}

\footnotetext{
${ }^{7}$ A titre d'exemple, rappelons que le secteur de la sidérurgie a perdu près de 8.000 emplois entre 1975 et la fin des années 1990, auxquels s'ajoutent la fermeture des chantiers navals en 1987 et la destruction de plusieurs milliers d'emplois à cette occasion.
} 


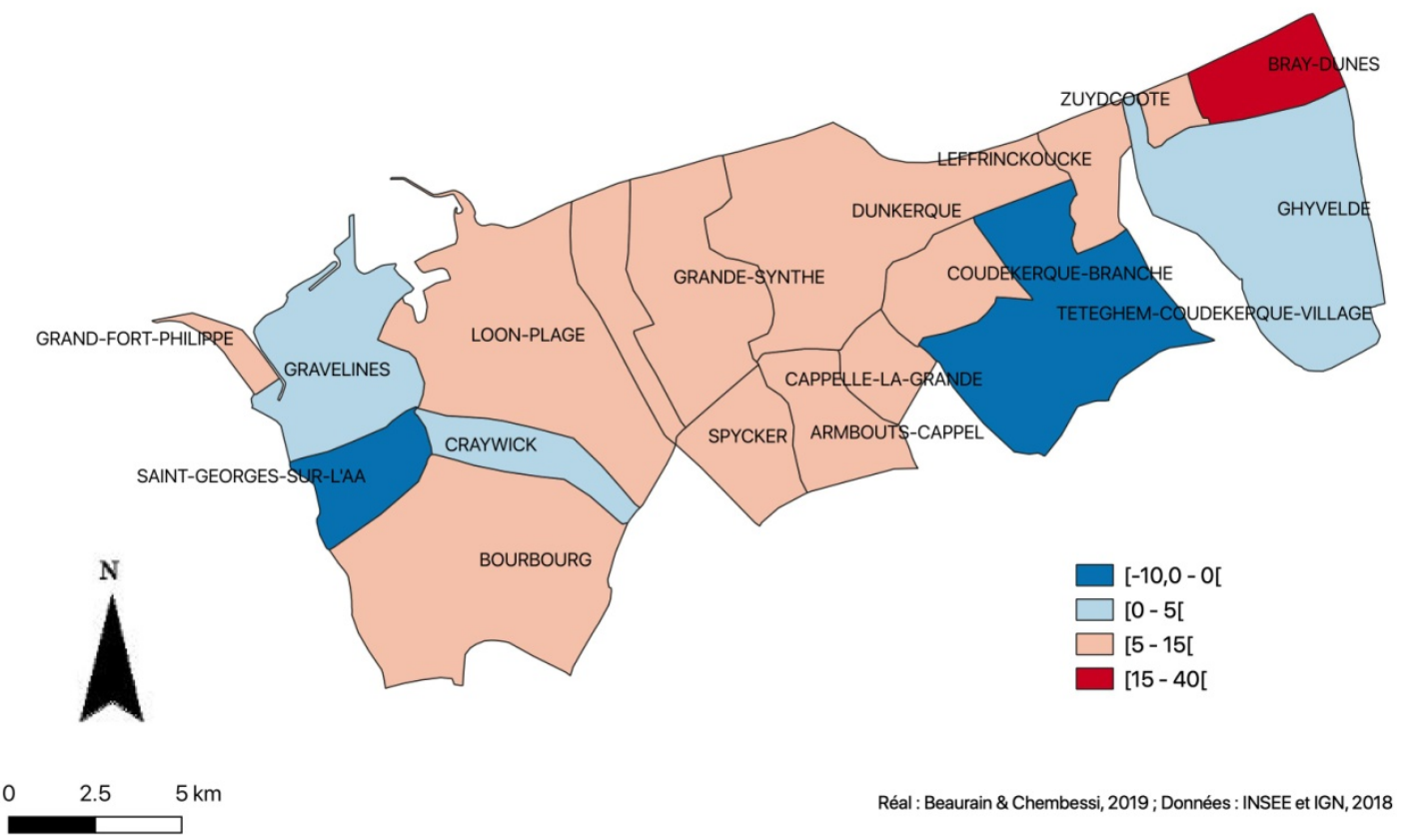

Il faut aussi souligner la multiplication des friches industrielles due à la disparation de quelques entreprises, notamment les chantiers navals en 1983. L'infrastructure urbaine est alors sous-exploitée ou progressivement à l'abandon, comme en témoigne l'amenuisement de l'offre commerciale de proximité (Clochey, 2017) ${ }^{8}$, autant de phénomènes que l'on peut observer dans des villes connaissant des formes de déclin plus ou moins prononcés (CauchiDuval et al., 2016). La diminution relative de l'activité industrielle de l'agglomération est à l'origine d'un processus de décroissance économique et démographique, quoique contrasté. Ce double déclin économique et démographique devient alors un enjeu essentiel de la réalité urbaine locale. Bien que Dunkerque soit un ancien bassin industriel touché par une désindustrialisation conséquente dans les années 1980, l'emploi local reste encore aujourd'hui fortement marqué par l'industrie.

De manière générale, la décroissance urbaine s'accompagne d'une réduction durable de l'effectif de population, et celle-ci semble soutenue par le passage à un nouveau régime démographique (Van de Kaa, 1987). Mais à Dunkerque, les transformations démographiques ne sont pas structurelles comme elles peuvent l'être dans la majorité des villes en décroissance. Ce paradoxe démographique s'explique par le fait que l'agglomération de Dunkerque, qui compte près de 200000 habitants, n'est pas soumise aux mêmes enjeux démographiques que la plupart des villes reconnues comme décroissantes en France (CauchiDuval et al., 2016). En effet, le taux de natalité, estimé à plus de 12,6\%o en 2015, est l'un des plus élevés de France. Et c'est davantage celui-ci qui expliquerait la récente reprise

\footnotetext{
${ }^{8}$ Une politique de rénovation urbaine active menée à partir des années 1990, concernant notamment la requalification des friches portuaires à proximité du centre ville a néanmoins permis de limiter ce phénomène.
} 
démographique, plutôt que les dynamiques migratoires. Ces dernières restent encore négatives avec un solde migratoire d'environ $-0,6 \%$ en 2015 , une situation que d'aucuns qualifient de " panne de croissance » puisque le solde naturel élevé compense à peine ce solde migratoire négatif. Le timide gain de population laisse d'ailleurs entrevoir des disparités entre les différentes communes de l'agglomération, tant en termes de dynamiques démographiques que sur le plan du profil socioprofessionnel de la population qui s'y installe (Clochey, 2017).

\section{Carte 4 : Taux de croissance démographique entre 2000 et 2018}

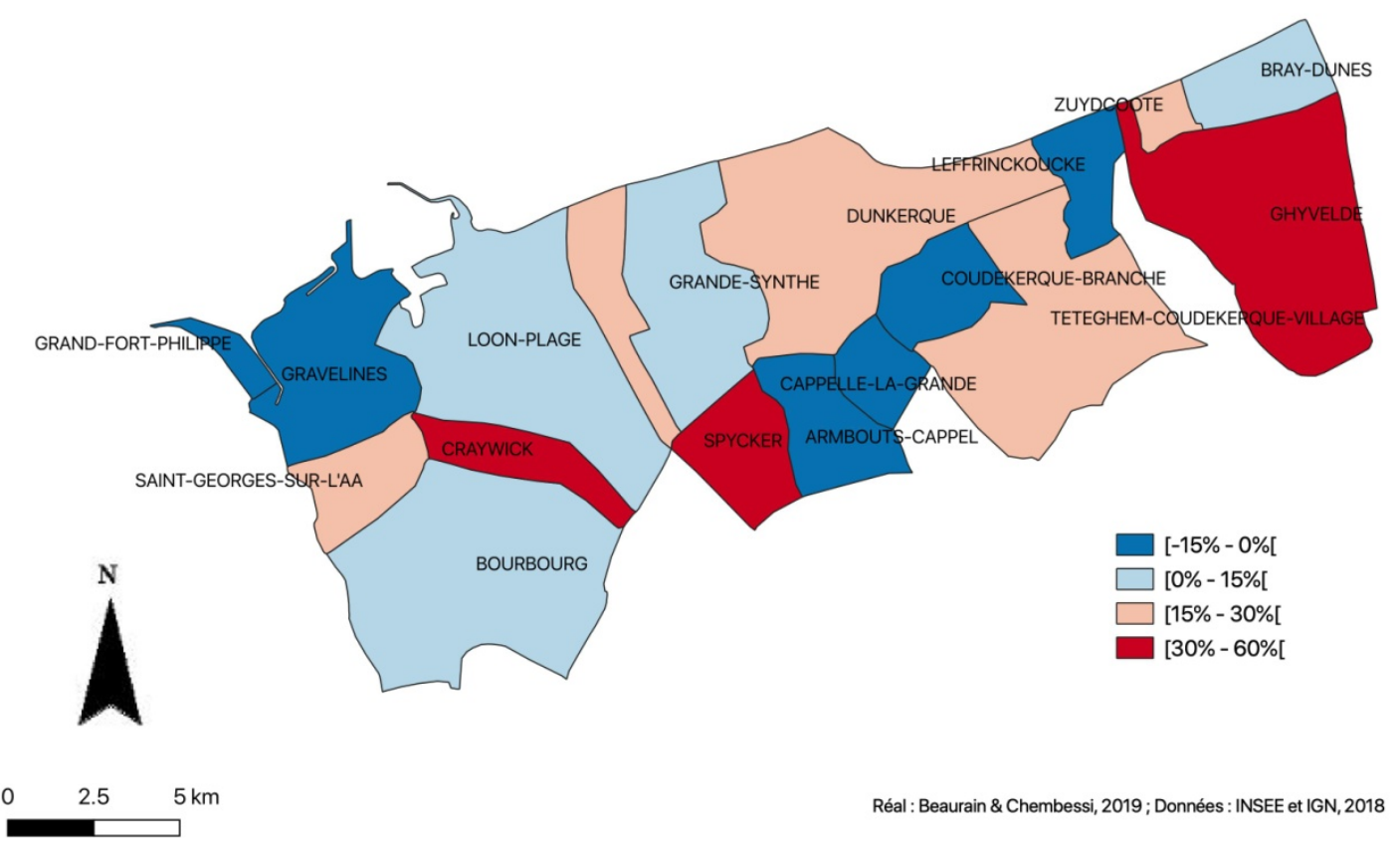

Les effets des crises économiques des années 1980, avec notamment la fermeture de plusieurs industries locales, se ressentent encore dans les communes de l'agglomération de manière plus ou moins intense. L'histoire urbaine de Dunkerque depuis cette période se rattache ainsi à la perception des villes en décroissance comme "des espaces urbains qui ont connu des pertes de population, un retournement économique, un déclin de l'emploi et des problèmes sociaux, symptômes d'une crise structurelle » (Martinez-Fernandez et al., 2012). Le cas de Dunkerque illustre bien le fait que la décroissance urbaine doit être considérée dans un ensemble diversifié et complexe, afin d'en appréhender les enjeux et/ou manifestations sur des villes moyennes. De plus, une reprise démographique peut masquer de possibles vulnérabilités du modèle urbain. La variation de la population - notamment si elle est considérée dans l'absolu - permet de résumer les dynamiques urbaines mais ne renseigne pas suffisamment sur la complexité du processus (Wolff et al., 2013), et encore moins sur le caractère multidimensionnel de celui-ci (Fol \& Cunningham-Sabot, 2010). La discontinuité de la décroissance ne saurait signifier l'absence de pertes relatives de population, de 
changements démographiques, d'un déficit d'attractivité notamment pour les jeunes, d'une réduction de l'activité économique voire d'un chômage structurel.

C'est dans ce contexte qu'à partir des années 1990, une politique de rénovation urbaine a permis d'améliorer sensiblement la qualité environnementale du territoire, répondant ainsi à une demande croissante des populations (Beaurain et al., 2017). En effet, les demandes croissantes des populations en faveur de l'amélioration de la qualité de vie ont incité au développement de politiques publiques visant à limiter les impacts environnementaux de l'industrie, notamment par le biais de la surveillance de la qualité de l'air et du développement d'une démarche d'écologie industrielle.

\section{Des synergies industrielles de Dunkerque}

Sur le territoire dunkerquois, les premiers échanges de flux de matières et d'énergie entre firmes qui apparaissent dans les années 1960 sont exclusivement liés au processus de production de l'entreprise sidérurgique dominante. Dès la mise en route de la production sidérurgique, en effet, s'est posée la question de la réutilisation du principal sous-produit de la production d'acier, le « laitier », difficilement stockable, sortant en grande quantité d'Usinor. C'est pour répondre à cette nécessité technique que va s'implanter en 1962 une société de traitement de résidus sidérurgiques au pied des hauts fourneaux, pour leur réutilisation dans la construction routière (Varlet, 2012). Parallèlement, Usinor va s'intéresser également à la valorisation des gaz issus de son process. L'implantation en 1963 d'une centrale thermique EDF à proximité du site répondra précisément à cet objectif, permettant au sidérurgiste de satisfaire ses besoins en électricité à partir de la chaleur fatale. Cette première phase, limitée à des acteurs privés, a pris un nouvel essor avec l'entrée en jeu de la Ville de Dunkerque en 1983, dans le cadre du projet de réutilisation de la chaleur fatale issue du process du sidérurgiste à destination du chauffage urbain. Le réseau ainsi créé atteindra près de $70 \mathrm{~km}$ à partir de 1985, en reliant les chaînes d'agglomération de la firme à des logements HLM, des bâtiments publics, puis plus récemment des logements privés ${ }^{9}$. La mise en place de cette synergie a eu des impacts significatifs sur les rejets de la firme sidérurgique dans l'atmosphère ( $\mathrm{CO}_{2}$ et poussières), tout en assurant le chauffage pour une partie de la population. Ce ne sont donc pas à proprement parler des raisons écologiques qui ont motivé la mise en place les premiers échanges de flux s'apparentant à de l'écologie industrielle. Ils l'ont été en raison des contraintes de process de production et des objectifs essentiellement économiques. L'intérêt écologique n'apparaît réellement qu'avec l'association des flux issus du process sidérurgique à un réseau de chauffage public.

Par ailleurs, la crise économique et la prise de conscience des problèmes environnementaux dans les années 1990 ont favorisé l'émergence d'une demande croissante de la population en faveur d'une amélioration de la qualité de vie et d'une réduction des impacts environnementaux de l'industrie. Pour répondre à cette demande, un véritable projet de territoire partagé va émerger au fil des documents de la planification territoriale, contribuant à

\footnotetext{
${ }^{9}$ Depuis 2010 , le réseau relie près de 30.000 équivalents logements.
} 
la construction d'une action collective autour de la notion d' " environnement industriel » (Beaurain, 2008). A l'absence de considération des entreprises pour toute valeur de l'environnement autre qu'économique, les populations locales ont ainsi progressivement opposé l'exigence d'un « air sain » sur le territoire. Les conflits, croissants et de plus en plus durs, entre les industriels, les habitants, et les représentants des associations de défense de l'environnement ont convaincu les autorités politiques locales de la nécessité d'engager des actions dans ce domaine (création d'un réseau de surveillance et de mesure de la qualité de l'air, création d'une structure de concertation, le Secrétariat pour la Prévention des Pollutions Industrielles, (SPPPI), et l'élaboration au début des années 2000 d'un Plan de Prévention de 1'Atmosphère (PPA)). Progressivement donc, un objectif commun s'est construit autour de la limitation des impacts sanitaires et la défense de la qualité de l'environnement.

Dans ce contexte territorial, de nouveaux projets relevant de l'écologie industrielle vont voir le jour. À partir du milieu des années 1980, de nouveaux échanges flux se mettent en place, principalement autour de l'activité sidérurgique : laitiers sidérurgiques, valorisation des gaz, chauffage urbain et valorisation de briques réfractaires. On observe à la fois un approfondissement des engagements entamés dès les années 1960 et l'émergence de nouvelles filières de valorisation (filières agricoles, des cimentiers, briques réfractaires...). La démarche connait alors un élargissement du réseau d'acteurs avec la création en février 2001 d'une structure chargée de promouvoir l'écologie industrielle, l'association ECOPAL (Economie et Ecologie, Partenaires dans l'Action Locale), à l'initiative des firmes les plus importantes du territoire et de certaines PME. Toujours dans le cadre des synergies industrielles, la centrale thermique EDF arrivant en fin de vie, une nouvelle centrale à cycle combiné d'une puissance de 790 MW, gérée par GDF-SUEZ, a été installée en 2005 sur le site de la firme sidérurgique. Plus d' $1 / 3$ de l'électricité produite par cette centrale est issue de la valorisation des gaz sidérurgiques, couvrant une très large partie des besoins du sidérurgiste, et les $2 / 3$ restants sont produits par la transformation du gaz naturel et vendus sur le marché de l'électricité via le réseau de transport d'électricité (RTE). De même, les cimentiers se sont aperçu que les coproduits issus de la fonte des hauts fourneaux pouvaient avoir des caractéristiques intéressantes et représentaient en outre une matière secondaire moins chère (Varlet, 2012).

Il apparaît ainsi que les gains résultant du développement de ces synergies industrielles ont pu être tout à fait significatifs pour l'ensemble des acteurs du territoire (Beaurain \& Varlet, 2015). Pour les entreprises, ont été constatées une baisse des coûts de production et une réduction de la quantité de déchets issue des processus de production, soit en leur donnant une seconde vie, soit en les utilisant à titre de combustibles en remplacement des ressources fossiles. Pour le territoire, cela a conduit à une réduction significative de la pollution atmosphérique, de la consommation des matières premières, et de la dépendance énergétique vis-à-vis des énergies non renouvelables. Le réseau de chauffage urbain constitue ainsi une source d'opportunités sociales pour le territoire en ce sens qu'il s'accompagne d'une réduction des coûts de distribution de la chaleur à destination des ménages locaux.

\section{De l'industrie au territoire: stratégie collective d'écologie industrielle et territoriale à Dunkerque}


L'analyse du processus de mise en œuvre des synergies industrielles révèle l'existence d'une mobilisation collective autour d'un enjeu non moins négligeable, celui d'associer la maîtrise du développement économique à la qualité environnementale locale. Ce que Beaurain et Brullot (2011) définissent comme une action collective en vue de résoudre un problème productif n'a été possible qu'en raison de l'appartenance des acteurs à un espace productif ou territorial commun (Brullot et al., 2014), mais aussi des capacités de coordination et de régulation des acteurs impliqués (Beaurain \& Varlet, 2015), et une activation progressive de l'action publique locale. Dans le cas de Dunkerque, le soutien public, notamment par l'engagement des partenariats publics-privés, a constitué un facteur déterminant dans l'acquisition d'une spécification locale, représentée ici par le déploiement territorial de l'ÉIT. En d'autres termes, celle-ci ne saurait être réduite aux échanges ou liens possibles entre acteurs économiques (ménages, associations, entreprises, etc.). Cela sous-entend aussi que les acteurs territoriaux se dotent d'une vision collective ou partagent des valeurs communes, permettant d'optimiser les ressources et les capacités dont ils disposent. Par conséquent, en dépit du potentiel du territoire, en termes de flux notamment, ce qui importe c'est la considération des expérimentations dans une logique territoriale intégrée, reposant sur un ensemble d'acteurs locaux, voire au-delà, afin de disposer d'un réel projet de développement territorial.

L'analyse de la démarche dunkerquoise apporte en outre des éléments intéressants sur la question de la diversité des valeurs en jeu dans le développement de l'économie circulaire. Si la préoccupation des entreprises est principalement économique, leur engagement s'effectue dans un contexte territorial marqué, on l'a dit, par l'importance des conflits d'usage à propos de la qualité de l'environnement. Ces conflits s'inscrivent globalement dans une volonté de restaurer la qualité de vie du territoire. Il ressort ainsi de l'expérience dunkerquoise une multiplicité d'intérêts en jeu : intérêts économiques pour les entreprises, intérêts environnementaux et sociaux pour les acteurs publics et les populations. Ces intérêts multiples renvoient manifestement à des valeurs différentes attachées par chaque acteur à la qualité de l'environnement. Si la valeur est clairement économique pour les entreprises (limiter le coût d'usage des ressources naturelles et dégager de nouveaux profits issus d'un usage différent de ces ressources), elle est motivée par d'autres références chez les acteurs publics et les populations. Ce constat suggère que les pratiques d'écologie industrielle ne peuvent s'appuyer sur une valeur unique et pose la question de la conciliation des multiples valeurs en jeu. En définitive, la démarche dunkerquoise révèle les jeux d'acteurs et formes d'action collective qui structurent ces synergies, et participent ainsi à la construction d'un territoire commun. Cette construction se situe à deux niveaux: dans la confrontation des valeurs entre les différents acteurs du territoire, d'une part; dans les interactions qui supportent les synergies industrielles d'autre part. Néanmoins, il faut souligner qu'à ce stade, l'engagement dans l'économie circulaire à Dunkerque, comme dans la plupart des autres villes françaises, reste pour une large part légitimé par un discours local en faveur d'un retour du territoire à la croissance économique. 
L'évolution positive en matière d'impacts environnementaux observée depuis le développement de la démarche d'écologie industrielle et territoriale ne doit pas masquer toutefois la situation encore largement défavorable du territoire dans ce domaine. Ainsi le Plan Climat Air Énergie 2015-2021 souligne-t-il par exemple que le territoire, zone de transit fortement industrialisée, avec un parc de logements anciens consommateurs d'énergie, émet encore 10 fois plus de gaz à effet de serre que la moyenne française. Dans la logique des implications de la problématique du réchauffement climatique, les axes stratégiques d'action proposés interpellent directement les principales composantes de la dynamique socioéconomique du territoire en appelant à un changement radical des comportements.

\section{L'ÉIT au service des territoires en difficulté}

\subsection{Le potentiel transformatif de l'écologie industrielle et territoriale}

L'exemple de l'agglomération dunkerquoise montre que les démarches d'écologie industrielle et territoriale, et au-delà sans doute d'économie circulaire, sont potentiellement porteuses de transformations profondes des pratiques industrielles et de stratégies de développement territorial. Pour autant, dans le discours politique comme dans le projet de territoire, elles ne se démarquent pas de manière nette des politiques classiques de recherche d'attractivité et de compétitivité des territoires. Il peut s'agir en effet simplement d'attirer les entreprises sur l'existence d'opportunités économiques (gains sur les coûts de production, sur le traitement des déchets...) et les populations, les plus sensibles à la question environnementale, par la mise en évidence d'une réelle volonté locale de mener des actions dans ce domaine. Dans ce cadre, ces démarches mises en œuvre au sein de villes industrielles en « panne de croissance » comme Dunkerque peuvent tout à fait s'inscrire dans l'idée d'une convergence possible de politiques de retour à la croissance industrielle et démographique et de politiques d'amélioration de la qualité environnementale. Les synergies industrielles se fondent alors sur une redynamisation territoriale par des politiques classiques de l'offre et de «croissance verte » dont on peut douter des effets réellement positifs sur la réduction de l'empreinte écologique des activités anthropiques. Ainsi, les impacts positifs pourraient-ils être largement annulés par des trajectoires de production et de consommation à la hausse, ce que la littérature économique définit sous le terme « d'effet rebond».

Néanmoins il ne faut pas négliger le potentiel transformatif porté par l'écologie industrielle et territoriale, à condition que les acteurs fassent le choix de l'insérer dans une véritable transition écologique. Nous rejoignons ici le propos développé par D. Bourg et C. Arnsperger, à travers le terme de « permacircularité ${ }^{10}$, pour qui l'enjeu essentiel en matière d'empreinte environnementale n'est pas tant celui des pollutions occasionnées par les activités anthropiques que celui, bien plus important, des quantités de flux (matières premières, énergie...). À ce titre, la permacircularité implique bien une politique volontariste de

\footnotetext{
${ }^{10}$ Le terme de permacircularité, ou d'« économie authentiquement circulaire » renvoie à l'approche proposée par la permaculture en agriculture, et indique que la « régénérativité de l'économie doit être perçue comme un principe englobant et s'appuyer sur une économie quasi auto-entretenue (Ansperger, Bourg, 2016)
} 
stagnation des volumes de production, calée sur les capacités de résilience de la planète et une réorganisation complète de l'économie. Il s'agit ainsi de prendre des initiatives concrètes dans l'optique d'une réduction significative des flux entrants et des consommations de ressources, pour produire et consommer moins (Arnsperger \& Bourg, 2017).

En d'autres termes, la permacircularité ne peut signifier simplement des engagements purement individuels d'entreprises, à un niveau micro. Elle doit constituer une trajectoire définie autour des actions et des contraintes collectives définies au niveau territorial et national. L'échelle macrosociétale aide à limiter les intrants de matières et d'énergie, ainsi qu'à développer une réflexion d'ensemble sur l'empreinte écologique des différents secteurs de l'économie (Arnsperger \& Bourg, 2016). En outre, dans cette logique de permacircularité et de forte limitation des volumes produits et consommés, la réflexion ne peut faire l'économie de l'évolution, depuis la fin de la croissance fordiste, du rôle de la technique et de « l'emploi-marchandise », et du sens du travail dans un système qui serait désormais régulé par la sobriété. Cela implique notamment une réflexion sur l'importance des circuits d'échange de proximité au sein des territoires et sur les motivations individuelles et collectives à "une sobriété volontaire" (Gorz, 2008). En ce sens, la permacircularité implique donc également des indicateurs qualitatifs qui permettent d'apprécier les transformations dans les modes de consommation mais également «la qualité du stock de savoir-faire et du degré de sens au travail » (Arnsperger \& Bourg, 2017). De ce point de vue, les nombreuses analyses mettant en exergue la nécessaire intégration des aspirations individuelles et collectives à la redécouverte de l'expérience du milieu naturel, y compris en milieu urbain, ouvrent des pistes de réflexion intéressantes. Dans le cas des villes en décroissance plus particulièrement, ces analyses soulignent par exemple l'importance des jardins urbains dans les politiques alternatives, tant du point de vue de la régénération des espaces en friche que de celui de la justice alimentaire (Chouraqui, 2017; Clochey, 2017; Paddeu, 2017), de la transition énergétique (Miot \& Rousseau, 2017 ; Florentin, 2017), etc.

Le potentiel transformatif d'une écologie industrielle et territoriale insérée dans une véritable transition écologique s'apprécie également à travers son impulsion donnée à la relocalisation de l'activité économique. En effet, elle oblige les entreprises à soumettre l'organisation de leurs process aux contraintes et aux opportunités locales. De plus, elle est potentiellement porteuse d'un développement de nouveaux secteurs économiques, à l'image de ceux relevant de l'économie sociale et solidaire par exemple, plus en phase avec les contraintes environnementales (Swaton, 2018). La transition énergétique, on l'a dit, offre la possibilité d'aller vers un tel ancrage territorial de la ressource. L'exemple de Dunkerque montre par exemple que, l'écologie industrielle et territoriale contribue à la relocalisation de la production énergétique, en se fondant sur la proximité géographique des producteurs et consommateurs concernés.

\subsection{Perspectives d'un nouveau contrat territorial au travers de l'ÉIT}

Les politiques alternatives de gestion des phénomènes de décroissance urbaine «laissent entrevoir l'émergence de nouvelles solidarités territoriales et/ou de redistribution spatiale 
allant à l'opposé des logiques de compétition » et fondées sur le principe d'égalisation territoriale (Florentin, 2017). La dimension alternative de l'écologie industrielle et territoriale peut également être appréciée au regard de ce «nouveau contrat social et territorial » dans le cadre de la décroissance urbaine. En effet, l'ÉIT se met au service du développement des entreprises ou acteurs économiques locaux (Hoffman et al., 2014), mais aussi d'un bien être territorial défini par d'autres critères que celui, classique, des richesses produites (JanyCatrice, 2016). De par sa capacité à générer de la «valeur territoriale » au travers d'un engagement collectif des acteurs territoriaux), l'écologie industrielle et territoriale renvoie à l'idée d'un ensemble de stratégies collectives d'adaptation locale aux crises. Dans le cas de Dunkerque, la valeur territoriale créée revêt principalement un caractère non marchand, renvoyant à une valeur sociale, collectivement construite, donc non dépendante d'une action individuelle (Maillefert \& Robert, 2017). On peut le constater avec la multitude d'acteurs locaux et nationaux qui participent au projet, notamment du point de vue d'un accompagnement technique et financier. La démarche permet d'intégrer les acteurs locaux dans un apprentissage collectif qui peut s'assimiler à une capacité de co-création et de coopération. De plus, elle permet de dépasser le cadre exclusif et restrictif de l'entreprise et de porter une vision globale pour le territoire, issue de la confrontation des différentes valeurs auxquelles les acteurs du territoire déclarent leur attachement. Comme indiqué précédemment, nous pensons que l'écologie industrielle et territoriale, qui repose sur l'engagement des entreprises dans une action collective territorialisée, constitue une illustration intéressante d'un «agir responsable » et d'une mission sociétale des entreprises, potentiellement au service de politiques alternatives. Dans le cas de Dunkerque, on l'a dit, les synergies inter-industrielles ont tout autant été justifiées par la réduction des coûts pour les entreprises, que par celle des impacts environnementaux pour le territoire et les populations les plus impactées par la pollution industrielle, et par la volonté de réduire les coûts d'accès au chauffage urbain pour les ménages les plus défavorisés.

Si l'expression de cette "valeur territoriale » renvoie à la réalisation d'un bénéfice partagé des politiques menées, elle implique également de manière fondamentale la question de la démocratie et de la participation des acteurs du territoire à la formulation du bien public. Dans une logique permacirculaire propre aux territoires s'inscrivant dans un chemin de limitation de croissance en effet, la prise en compte de la contrainte environnementale implique des pratiques de concertation et d'action collective entre des acteurs (population, entreprises, acteurs publics). Et dans bien des cas, ces acteurs locaux n'ont pas nécessairement l'habitude d'interagir. La question à traiter devient alors celle des modalités de cette interaction.

\section{Conclusion}

Parce qu'elles sont frappées de plein fouet par des situations de crise socio-économique et écologique, les villes en décroissance constituent des «laboratoires" intéressants pour l'émergence de nouvelles politiques publiques destinées à faire face à ce déclin et porteuses d'un redéveloppement alternatif. L'enjeu de la «décroissance» comme trajectoire d'évolution n'a jusqu'à présent guère été formulé au niveau territorial, à l'exception des argumentaires s'inscrivant d'emblée dans le «post-capitalisme ». L'écologie industrielle et 
territoriale, et au-delà l'économie circulaire, offre précisément selon nous des opportunités pour la mise en œuvre de politiques publiques potentiellement constitutives d'un modèle de développement alternatif. Comme le montre l'exemple de l'agglomération dunkerquoise, ville frappée depuis les années 1980 par un déclin démographique et industriel à peine compensé par des évolutions récentes un peu plus positives, la mise en œuvre d'une démarche d'écologie industrielle implique en effet une réflexion en profondeur sur les flux constitutifs du système productif local. L'évolution dans le temps des synergies industrielles, ainsi que le projet de territoire esquissé au fil des documents de la planification territoriale, révèlent l'émergence progressive et la consolidation d'une action collective portée par des acteurs privés et publics. Celle-ci est par ailleurs alimentée par des revendications sociales en faveur d'une amélioration de la qualité environnementale. Pour autant, des ambiguïtés demeurent quant au positionnement de cette action collective au regard des politiques d'inspiration néolibérale de développement territorial. En effet, l'écologie industrielle et territoriale, en tant que nouvelle trajectoire de développement pour les villes en décroissance, n'est pas nécessairement synonyme d'une véritable limitation des flux de matière et d'énergie et peut, paradoxalement, s'inscrire dans un regain de croissance économique, pouvant accroître l'empreinte écologique. Cela indique probablement que la «décroissance» comme fondement d'un projet de territoire implique bien des contraintes supplémentaires, notamment au niveau de la planification territoriale, pour se rapprocher de la permacircularité, ainsi qu'une réflexion collective sur la valeur territoriale de ces démarches.

\section{Bibliographie}

ADEME. (2017). Économie Circulaire: un atout pour relever le défi de l'aménagement durable des territoires. (Livre blanc) (p. 116).

Allenby, B. (1992). Design for environment: implementing industrial ecology ( $\mathrm{PhD}$ Dissertation). Rutgers University, New Brunswick, NJ.

Angot, S. (2013). « Plans climat-énergie territoriaux et Agenda 21. Des outils au service de la transition?» Mouvements, 75, automne, 125-134

Arnsperger, C., Bourg, D. (2016). « Vers une économie authentiquement circulaire. » Revue de l'OFCE, (145), 91-125.

Arnsperger, C., Bourg, D. (2017). Écologie intégrale : pour une société permacirculaire (1re édition). Paris.

Aurez, V., Georgeault L. (2016a). Les indicateurs de l'économie circulaire en Chine. Revue de l'OFCE (145), 127-160.

Aurez, V., Georgeault L. (2016b). Économie circulaire : système économique et finitude des ressources. Louvain-la-neuve. De Boeck.

Béal, V., Fol, S., Rousseau, M. (2016). « De quoi le «smart shrinkage» est-il le nom ? Les ambiguiités des politiques de décroissance planifiée dans les villes américaines. » Géographie, économie, société, 18(2), 211-234.

Béal, V., \& Rousseau, M. (2014). «Alterpolitiques! » Métropoles, (15). Consulté à l'adresse http://journals.openedition.org/metropoles/4948 
Beaurain, C., Andrieu, M. (2016). « La ville durable comme fard de la ville néolibérale. Penser l'urbain d'aujourd'hui sans œillères. " Revue du MAUSS permanente. http://www.journaldumauss.net/./?La-ville-durable-comme-fard-de-la

Beaurain, C., Brullot, S. (2011). « L'écologie industrielle comme processus de développement territorial : une lecture par la proximité. » Revue d'économie régionale et urbaine, (2), page 313-340.

Beaurain, C., Maillefert, M., Varlet, D. (2017). « La proximité au cœur des synergies écoindustrielles dunkerquoises. » Flux, (109-110), 23-35.

Beaurain, C., Varlet, D. (2015). « Régulation des interactions au sein d'un réseau territorialisé d'entreprises dans le cadre de l'écologie industrielle. » Revue d'économie industrielle, (152), 173-206.

Beaurain, C. (2008). « La construction d'un territoire à partir des ressources environnementales: l'exemple de l'agglomération dunkerquoise », Géographie, Economie, Société, vol. 10, pp. 365-384.

Bocken, N., Short, S., Rana, P., \& Evans, S. (2014). « A literature and practice review to develop sustainable business model archetypes. » Journal of Cleaner Production, 65, 42-56.

Bost, F. (2014). La France : mutations des systèmes productifs. Paris : Armand Colin.

Bourg, D. (2012). Transition écologique plutôt que développement durable. Vraiment Durable, 1(1), 77-96

Brullot, S., Maillefert, M., \& Joubert, J. (2014). « Stratégies d'acteurs et gouvernance des démarches d'écologie industrielle et territoriale. " Développement durable et territoires. Économie, géographie, politique, droit, sociologie, (Vol. 5, $\mathrm{n}^{\circ} 1$ ). https://doi.org/10.4000/developpementdurable.10082

Buclet, N. (2015). Écologie Industrielle et économie circulaire : définitions et principes. In Économie circulaire et écosystèmes portuaires. (Yann Alix ; Nicolas Mat ; Juliette Cerceau, p. 27-41). Éditions EMS, Management \& Société.

Castells, M., Godard F. (1974), Monopolville. L'entreprise, l'Etat, l'urbain. Paris : Mouton.

Cauchi-Dubal, N., Béal, V., Rousseau, M. La décroissance urbaine en France : des villes sans politique », Espace populations sociétés [En ligne], 2015/3-2016/1 | 2016, mis en ligne le 20 mars 2016, URL : http://journals.openedition.org.acces.bibl.ulaval.ca/eps/6112

Charmes, E. (2011), La ville émiettée. Essai sur la clubbisation de la vie urbaine, Paris, PUF. Chouraqui, J. (2017). « Dunkerque, où construire et pour qui ? "Revue Sur-Mesure, ([En Ligne], 3), mis en ligne le 26/04/2018, URL: http://www.revuesurmesure.fr/issues/dunkerque-ou-construire-et-pour-qui

Clerc, D. (2004). « De l'état stationnaire à la décroissance: histoire d'un concept flou. » Alternatives Économiques/L'économie politique, 2(22), 76-96.

Clochey, L. (2017). Politiques alternatives top down en territoire industriel : quelle portée pour les jardins populaires? Cas de Grande-Synthe (59) (Mémoire de Master). Université Paul Valery Montpellier 3.

Cunningham-Sabot, E., Roth, H. (2013). « Growth Paradigm against Urban Shrinkage: A Standardized Fight? The Cases of Glasgow (UK) and Saint-Étienne (France). »In 
Shrinking Cities - International Perspectives and Policy Implications (Pallagast K., Martinez-Fernandez C., Wiechmann T. (eds), p. 99-124). New York: Routledge.

Daly, H., Farley, J. (2010). Ecological Economics, Second Edition: Principles and Applications (Second edition). Washington, DC: Island Press.

Ehrenfeld, J. (2004). «Industrial ecology: a new field or only a metaphor? » Journal of Cleaner Production, 12(8), 825-831.

Erkman, S. (1997). " Industrial ecology: An historical view. " Journal of Cleaner Production, 5(1), 1-10.

Erkman, S. (2004). Vers une écologie industrielle (2e édition). Paris : Ed. Charles Léopold Mayer.

Florentin D., «Des réseaux qui décroissent, des solidarités qui s'accroissent? Baisse des consommations d'eau et d'énergie et nouveau contrat social et territorial», Métropolitiques, 17 avril 2017. URL : https://www.metropolitiques.eu/Des-reseauxqui-decroissent-des.html

Fol, S., Cunningham-Sabot, E. (2010). « «Déclin urbain » et Shrinking Cities: une évaluation critique des approches de la décroissance urbaine. » Annales de géographie, (674), 359-383.

Gadrey, J. (2015). Adieu à la croissance: bien vivre dans un monde solidaire (Troisième édition augmentée). Paris: Alternatives économiques.

Geddes, P. (1915). Cities in evolution: an introduction to the town planning movement and to the study of civics. London: Williams.

Ghisellini, P., Cialani, C., \& Ulgiati, S. (2016). « A review on circular economy: the expected transition to a balanced interplay of environmental and economic systems. » Journal of Cleaner Production, 114, 11-32.

Gorz, A. (2008). Ecologica. Paris: Galilée.

Hampikian, Z. (2017). « Distribuer la chaleur fatale des entreprises: la construction dynamique d'un réseau d'énergie décarbonée, entre flux et infrastructure. " Développement durable et territoires. Économie, géographie, politique, droit, sociologie, (Vol. 8, n²). https://doi.org/10.4000/developpementdurable.11736

Harvey, D. (2004). «L'urbanisation du capital. » Actuel Marx, 35 (1), 41-70.

Harvey, D. (2010). The enigma of capital: and the crises of capitalism. New York : Oxford University Press.

Hatchuel, A., Segrestin, B. (2007). « La société contre l'entreprise ? Vers une norme d'entreprise à progrès collectif. » Droit et société. 1 (65). 27-40

Hatchuel, A., Querrien, A. (2018). «L'entreprise à mission et ses partenaires. » Multitudes. 3 (72), 211-217

Hatchuel, A., Segrestin, B. (2016). " Trois propositions pour fonder une entreprise convivialiste. » Revue du MAUSS, 2 (48), 165-172

Hatchuel, A., Levillain, K., Segrestin, B., Varnac, K. (2014). La société à objet social étendu : un nouveau statut pour l'entreprise. Paris, Presses des MinesHobson, K. (2016). « Closing the loop or squaring the circle? Locating generative spaces for the circular economy. » Progress in Human Geography, 40 (1), 88-104.

Hoffman, J., Corbett, J., Joglekar N., \& Wells, P. (2014). «Industrial Ecology as a Source of Competitive Advantage. » Journal of Industrial Ecology, 18(5), 597-602. 
Hopkins, R. (2010). Écosociété | Manuel de Transition. De la dépendance au pétrole à la résilience locale.

Howard, E. (1902). Garden Cities of Tomorrow. Swan Sonnenschein \& Co., Ltd.

Jackson, T. (2009). Prosperity without growth. Economics for a Finite Planet. London. Sterling : Earthscan.

Jany-Catrice, F. (2016). "La mesure du bien-être territorial. » Revue de l'OFCE, (145), 63-90.

Jelinski, L., Graedel, E., Laudise, A., McCall, W., \& Patel, K. (1992). « Industrial ecology: concepts and approaches. " Proceedings of the National Academy of Sciences of the United States of America, 89(3), 793-797.

Joan, J.-M., Méha, C., Hellequin, A.-P., \& Beaurain, C. (2017). Genèse d'un territoire industrialo-portuaire. In H. Flanquart \& S. Frère (Éd.), La ville et ses risques : Habiter Dunkerque (p. 25-42). Villeneuve d'Ascq : Presses universitaires du Septentrion. 2

Korhonen, J. (2004). « Theory of industrial ecology. » Progress in Industrial Ecology, an International Journal, 1(1-3), 61-88.

Laigle, L. (2013). « Pour une transition écologique à visée sociétale. 》 Mouvements, 75, automne, 135-141

Lilley, D. (2009). « Design for sustainable behaviour: strategies and perceptions. » Design Studies, 30(6), 704-720.

Lyle, J. (1994). Regenerative design for sustainable development. New York: John Wiley.

Magnaghi, A. (2003). Le projet local. Sprimont, Belgique : P. Mardaga.

Magnaghi, A. (2014). La biorégion urbaine : petit traité sur le territoire bien commun. Paris : Eterotopia France.

Maillefert, M., \& Robert, I. (2017). « Nouveaux modèles économiques et création de valeur territoriale autour de l'économie circulaire, de l'économie de la fonctionnalité et de l'écologie industrielle. \Revue d'Économie Régionale \& Urbaine, Décembre (5), 905-934.

Martinez-Fernandez C., Audirac I., Fol S., Cunningham-Sabot E., 2012, « Shrinking Cities : UrbanChallenges of Globalization », International Journal of Urban and Regional Research, 36 (2), 213-225.

Meadows, D., \& Randers, J. (2012). Les Limites à la croissance ( $1^{\text {re }}$ éd.). Paris : Rue de l'échiquier.

Miot, Y., Rousseau M., (2017) «Décroître pour survivre? Démolitions et transition énergétique à Vitry-le-François », Métropolitiques, 29 juin 2017. URL : https://www.metropolitiques.eu/Decroitre-pour-survivre.html

Montel, O. (2017). "La décroissance: une utopie? » Les Cahiers français (Paris. 1956), (401), p.57-65.

Mumford, L. (1964). La cité à travers l'histoire. Seuil.

Mumford, L. (2016). Technique et civilisation. Marseille : Parentheses.

North, P., Nurse, A. (2014). « Beyond entrepreneurial cities. Towards a post-capitalist grassroots urban politics of climate change and resource constraint. " Métropoles, (15). http://journals.openedition.org/metropoles/5005

Paddeu, F. «Sortir du mythe de la panacée. Les ambiguïtés de l'agriculture urbaine à Détroit »,

Métropolitiques,

13

avril

2017. 
URL : https://www.metropolitiques.eu/Sortir-du-mythe-de-la-panacee-Lesambiguites-de-1-agriculture-urbaine-a-Detroit.html

Paulus, F. (2004) Coévolution dans les systèmes de villes: croissance et spécialisation des aires urbaines françaises de 1950 à 2000. Thèse de Doctorat, Géographie Université Panthéon-Sorbonne - Paris I, 409p.

Preston, F. (2012). A Global Redesign? Shaping the Circular Economy (Briefing paper) (p. 20). London: Chatham House Breifing Paper.

Raineau, L. (2011). «Vers une transition énergétique?», What can we expect from renewable energies? » Natures Sciences Sociétés, 19(2), 133-143.

Rocher, L. (2013). « Le chauffage urbain dans la transition énergétique : des reconfigurations entre flux et réseau, District heating and energy transition : reconfigurations between flow and network. » Flux, (92), 23-35.

Rousseau, M., \& Béal, V. (2015). " Alterpolitiques! 2. " Métropoles, (17). http://journals.openedition.org/metropoles/5146

Swaton, S. (2018). Pour un revenu de transition écologique. Paris : Presses Universitaires de France - PUF.

Van de Kaa, D. (1987), «Europe's Second Demographic Transition », Population Bulletin, vol. 42 , pp. 1-57.

Varlet, D. (2012). Enjeux, potentialités et contraintes de l'écologie industrielle: le cas de Dunkerque, Thèse de doctorat, Sciences de gestion, Université du Littoral Côté d'Opale, Dunkerque.

Wolff, M., Fol, S., Roth, H., \& Cunningham-Sabot, E. (2013). « Shrinking Cities, villes en décroissance : une mesure du phénomène en France. » Cybergeo : European Journal of Geography. 